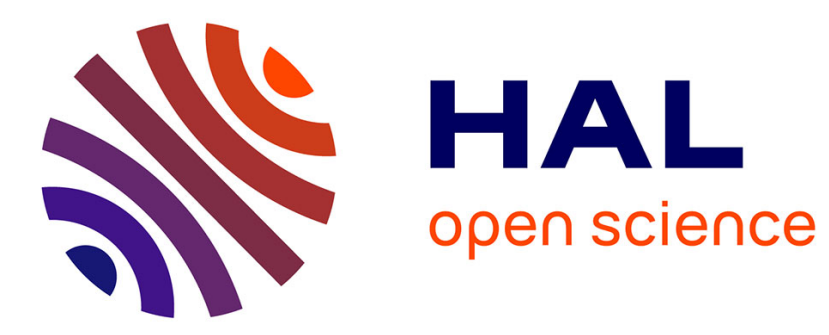

\title{
Validation d'une méthode qualitative d'estimation de l'influence de la météorologie sur le bruit
}

\author{
V. Zouboff, Y. Brunet, E. Sechet, J. Bertrand
}

\section{To cite this version:}

V. Zouboff, Y. Brunet, E. Sechet, J. Bertrand. Validation d'une méthode qualitative d'estimation de l'influence de la météorologie sur le bruit. Journal de Physique IV Proceedings, 1994, 04 (C5), pp.C5-813-C5-816. 10.1051/jp4:19945175 . jpa-00252856

\section{HAL Id: jpa-00252856 https://hal.science/jpa-00252856}

Submitted on 1 Jan 1994

HAL is a multi-disciplinary open access archive for the deposit and dissemination of scientific research documents, whether they are published or not. The documents may come from teaching and research institutions in France or abroad, or from public or private research centers.
L'archive ouverte pluridisciplinaire HAL, est destinée au dépôt et à la diffusion de documents scientifiques de niveau recherche, publiés ou non, émanant des établissements d'enseignement et de recherche français ou étrangers, des laboratoires publics ou privés. 


\title{
Validation d'une méthode qualitative d'estimation de l'influence de la météorologie sur le bruit
}

\author{
V. ZOUBOFF, Y. BRUNET* ${ }^{*}$ E. SECHET et J. BERTRAND \\ Laboratoire des Ponts \& Chaussées d'Angers, BP. 69, 49136 Les Ponts de Ce, France \\ * INRA Bioclimatologie, Bordeaux, France
}

\begin{abstract}
We first propose a simple method for helping the acoustician to estimate the effects of meteorological conditions on noise propagation, at least qualitatively. This method is based on a double-entry grid that only requires simple meteorological observations. We then compare the qualitative estimations given by the grid to the results of an outdoor experiment. Human evaluation of climatic conditions is shown to be satisfactory for a qualitative estimation of their effects on noise measurements.
\end{abstract}

\section{1 - INTRODUCTION}

Cette communication présente une validation expérimentale d'une méthode simple, destinée aux acousticiens de terrain, visant à prendre en compte qualitativement les effets météo sur des mesures acoustiques effectuées à grande distance d'une source.

Dans un premier temps, on rappelle le principe et le mode d'utilisation de cette méthode. On montre ensuite sa validité par confrontation à des données expérimentales.

\section{2 - PRESENTATION DE LA METHODE}

Toutes les études sur le sujet ont montré que les gradients de température et de vecteur vent sont les facteurs prépondérants dans l'influence des conditions atmosphériques sur la propagation du son. Des analyses factorielles et des classifications hiérarchiques sur des données expérimentales obtenues sur le site de La Crau, nous ont permis d'une part de confirmer ces effets prépondérants, et d'autre part, d'identifier et de caractériser les variables météorologiques qui sont à f'origine des formes des gradients [1]. En définitive, on obtient une grille à double entrée ( $\mathrm{Ti}$ : conditions thermiques $/ \mathrm{Ui}$ : conditions aérodynamiques) dont on présente dans [2] et [3] la méthodologie complète de mise au point.

$$
\text { Les conditions d'entrée ( Ui ; Ti) de la grille répondent aux critères suivants : }
$$

\begin{tabular}{|c|c|c|c|}
\hline U1 : & $\begin{array}{l}\text { Vent fort ( } 3 \text { à } 5 \mathrm{~m} / \mathrm{s} \text { ) contraire au sens } \\
\text { émetteur-récepteur } \\
\text { vent moyen à faible }(1 \text { à } 3 \mathrm{~m} / \mathrm{s} \text { ) } \\
\text { contraire ou vent fort, peu contraire } \\
\text { vent nul ou vent quelconque de } \\
\text { travers } \\
\text { vent moyen à faible portant ou vent } \\
\text { fort peu portant }\left(\approx 45^{\circ}\right) \\
\text { vent fort portant }\end{array}$ & T3: & $\begin{array}{l}\text { sèche et peu de vent } \\
\text { mêmes conditions que T1 mais au } \\
\text { moins une est non vérifiée } \\
\text { lever du soleil ou coucher du soleil } \\
\text { ou (temps couvert et venteux et } \\
\text { surface pas trop humide) } \\
\text { Nuit et ( nuageux ou vent) } \\
\text { Nuit et ciel dégagé et vent faible }\end{array}$ \\
\hline
\end{tabular}


L'estimation de la classe météo (Ui;Ti) permet de fournir un ordre de grandeur des effets des gradients sur la propagation sonore, par l'intermédiaire de la grille ci-dessous:

On peut y distinguer cinq types de propagation, en deux groupes dont les effets sont asymétriques:

- - : Atténuation très forte

-: Atténuation moyenne

$\mathbf{Z}$ : effets météo nuls

+ : renforcement très faible

++ : renforcement moyen

(les cas notés sont exclus )

Par exemple, la condition d'entrée U3 caractérise une influence aérodynamique nulle, et $T 3$, une influence thermique nulle. Les classes U1T2 ou U5T4 correspondent à des situations de synergie des effets des gradients, alors que U2T4 ou U4T2 à des situations de compensation.

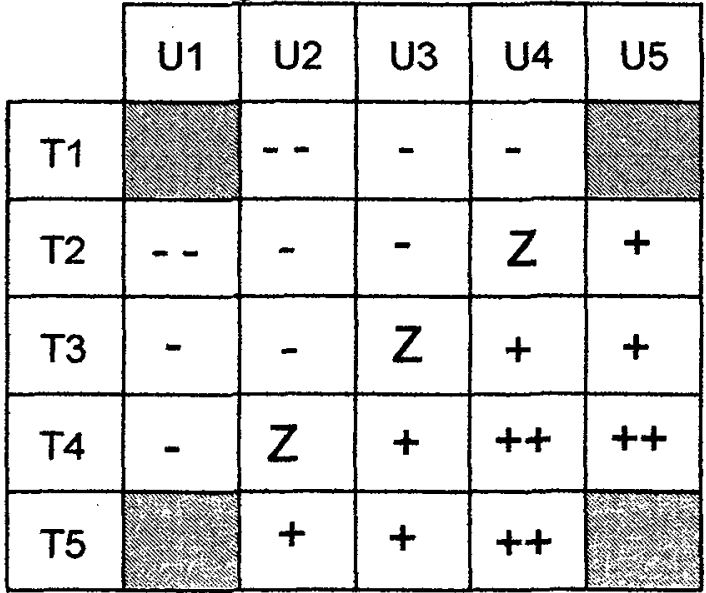

Fig. 1 : grille UiTi

A l'aide de cette grille, il est possible de comparer qualitativement plusieurs mesures acoustiques réalisées dans des conditions météorologiques différentes. On s'aperçoit aussi que certaines conditions météo sont plus propices à des mesures acoustiques que d'autres.

\section{3 - DISPOSITIF EXPERIMENTAL DE VALIDATION}

Cette validation expérimentale a été effectuée sur un site d'Alsace, donc avec des effets de sol différents du site de La Crau qui a permis la mise au point de la grille. Le dispositif expérimental est résumé par le schéma suivant:

Simultanément aux mesures acoustiques, des opérateurs ont estimé les classes météo UiTi . Ces estimations "au jugé", ont été comparées à des mesures issues de capteurs météo placés à différents niveaux d'un mât de $10 \mathrm{~m}$, à un endroit représentatif de la propagation; Mais en aucun cas, ces opérateurs n'ont eu recours aux valeurs numériques des capteurs.

Dans l'ensemble, les estimations furent relativement fiables.

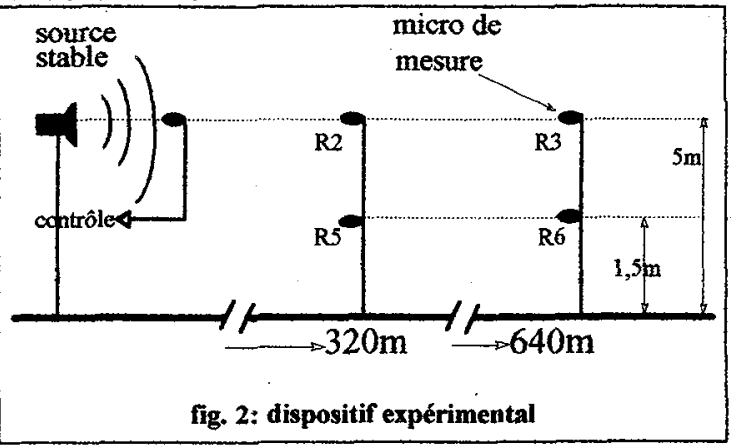

\section{4 - PROJECTION D'UNE CHRONOLOGIE}

On se propose ici de valider la grille par la projection d'échantillons de très courte durée (10 $\mathrm{min})$, et de montrer qu'une évolution dans la grille s'accompagne d'une progression des niveaux sonores correspondants.

On considère une suite d'échantillons de 10 minutes, formant une chronologie sur un après midi, de $14 \mathrm{~h} 00$ à $16 \mathrm{~h} 30$. Toutes les 10 minutes, un opérateur ré-évalue les paramètres d'entrée UiTi de la grille.

Une simple analyse graphique montre que les quatre récepteurs sont globalement très corrélés.

L'examen des mesures météo effectuées simultanément, nous indique que les conditions atmosphériques sont restées stables durant tout l'après midi, mis à part la direction du vent. De 14 h00 a 15 h20 environ, le vent reste entre "travers" et "contraire". Puis il devient 
nettement "de travers", pour enfin passer au "portant" vers $15 \mathrm{~h} 40$. Il revient au "contraire" vers $16 \mathrm{~h} 00$ et suit la même évolution vers le portant mais sur $1 / 2$ heure, soit jusqu'à $16 \mathrm{~h} 30$.

Le schéma de la figure 3 projette sur une partie localisée de la grille, les niveaux équivalents

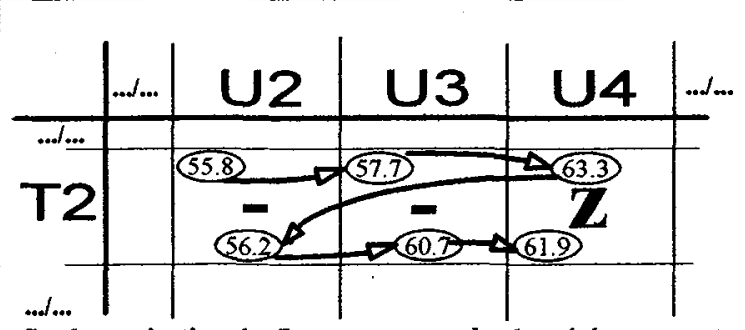
par tranche de même classe UiTi , au récepteur $\mathrm{R} 3(640 \mathrm{~m}, 5 \mathrm{~m})$.

On s'aperçoit que l'évolution vers une propagation de moins en moins défavorable,

(i.e. rapprochement du $\mathbf{Z}$ ) s'accompagne d'une croissance du niveau sonore.

fig. 3 : projection des $L_{A}$ eq par tranche de météo constante

L'évolution des paramètres UiTi de la grille, correspond à la perception qu'a l'opérateur des variations atmosphériques. On s'aperçoit donc que cette perception, bien qu'imparfaite, est suffisamment fiable pour en estimer à l'aide de la grille les incidences qualitatives sur la propagation acoustique.

\section{5: PROJECTIONS D'ECHANTILLONS DE DUREE MOYENNE}

Les échantillons représentent ici des intégrations de 30 minutes à 3 heures, qui correspondent aux périodes pendant lesquelles les conditions atmosphériques sont restées globalement stables.

Pour chacun des échantillons, un opérateur a estimé les caractéristiques Ui \& Ti suivant les critères définis plus haut, sans avoir recours aux instruments de mesure du mât météo. Ces estimations sont établies de façon à être représentatives des conditions atmosphériques de la période d'intégration.

Le tableau $n^{\circ} 1$ présente pour chaque échantillon, sa durée d'intégration, sa localisation dans la journée, les niveaux sonores $L_{A}$ eq mesurés à $320 \mathrm{~m}$ et $640 \mathrm{~m}$ de la source, et la classe météo UiTi estimée par l'opérateur.

\begin{tabular}{|c|c|c|c|c|c|c|c|c|}
\hline & \multicolumn{8}{|c|}{ Tableau 1 : description des échantillons } \\
\hline \multirow{2}{*}{$\begin{array}{c}\mathrm{n}^{\circ} \\
\text { tranche }\end{array}$} & \multicolumn{2}{|c|}{ (heure légale) } & \multirow[b]{2}{*}{ durée } & \multirow{2}{*}{$\begin{array}{c}320 \mathrm{~m} \\
5 \mathrm{~m} \\
\mathbf{R 2}\end{array}$} & \multirow{2}{*}{$\begin{array}{c}320 \mathrm{~m} \\
1.5 \mathrm{~m} \\
\text { R5 }\end{array}$} & \multirow{2}{*}{$\begin{array}{c}640 \mathrm{~m} \\
5 \mathrm{~m} \\
\mathrm{R3}\end{array}$} & \multirow{2}{*}{$\begin{array}{c}640 \mathrm{~m} \\
1.5 \mathrm{~m} \\
\mathrm{R} 6\end{array}$} & \multirow{2}{*}{$\begin{array}{l}\text { classe météo } \\
\text { estimée }\end{array}$} \\
\hline & début & fin & & & & & & \\
\hline 1 & $15: 25: 00$ & $18: 25: 00$ & $3 \mathrm{~h}$ & 71.1 & 60.4 & 54.7 & 50.7 & U2-T2 \\
\hline 2 & $09: 40: 00$ & $10: 59: 50$ & $1 \mathrm{~h} 20^{\prime}$ & 76.5 & 67.9 & & 56.0 & U4-T2 \\
\hline 3 & $13: 55: 00$ & $15: 44: 50$ & $1 \mathrm{~h} 50^{\prime}$ & 77.0 & 69.6 & 64.1 & 59.3 & U4-T2 \\
\hline 4 & $19: 55: 00$ & $20: 35: 00$ & $40^{\prime}$ & 72.7 & 73.5 & 64.5 & 64.4 & U4-T3 ou U3-T3 \\
\hline 4 & $20: 35: 00$ & $21: 15: 00$ & $40^{\circ}$ & 73.6 & 75.9 & 65.2 & 65.4 & U3-T5 \\
\hline 4 & $21: 15: 00$ & $21: 55: 00$ & $40^{\prime}$ & 76.6 & 77.1 & 66.9 & 65.3 & U3-T5 \\
\hline 4 & $21: 55: 00$ & $22: 35: 00$ & $30^{\circ}$ & 74.1 & 76.1 & 66.2 & 64.7 & U3-T5 \\
\hline 5 & $10: 10: 00$ & $10: 50: 00$ & $40^{\prime}$ & 74.8 & 65.8 & 61.4 & 55.5 & U4-T2 ou U3-T2 \\
\hline 5 & $10: 50: 00$ & $11: 20: 00$ & $30^{\prime}$ & 73.1 & 63.4 & 58.8 & 53.5 & U2-T2 \\
\hline 5 & $11: 20: 00$ & $11: 59: 50$ & $40^{\prime}$ & 69.2 & 60.2 & 57.3 & 53.3 & U2-T2 \\
\hline 6 & $15: 30: 10$ & $16: 40: 00$ & $1 \mathrm{~h} 10^{\prime}$ & 69.0 & 61.1 & 57.2 & 54.3 & $\mathrm{U} 2-12$ \\
\hline 7 & $09: 15: 40$ & $10: 55: 00$ & 1h40 & 75.4 & 66.1 & 60.1 & 54.7 & U3-T2 \\
\hline 8 & $14: 00: 10$ & $14: 40: 00$ & $40^{\prime}$ & 68.3 & 58.8 & 52.1 & 55.7 & U2-T2 \\
\hline 8 & $14: 40: 00$ & $15: 30: 00$ & $50^{\prime}$ & 70.6 & 61.1 & 52.5 & 56.6 & U2-T2 \\
\hline 8 & $15: 30: 00$ & $16: 29: 50$ & $1 \mathrm{~h}$ & 73.3 & 64.7 & 55.9 & 61.3 & $\mathrm{U} 4-\mathrm{T} 2$ \\
\hline
\end{tabular}

On constate sur le tableau 1 que ce sont évidemment les récepteurs à $640 \mathrm{~m}$ qui sont les plus sensibles aux conditions météorologiques. En projetant sur la grille par exemple les niveaux sonores obtenus au point R3 (donc à $640 \mathrm{~m}$ de la source et à $5 \mathrm{~m}$ du sol), on constate une bonne concordance des mesures avec les prévisions (fig. 4) 


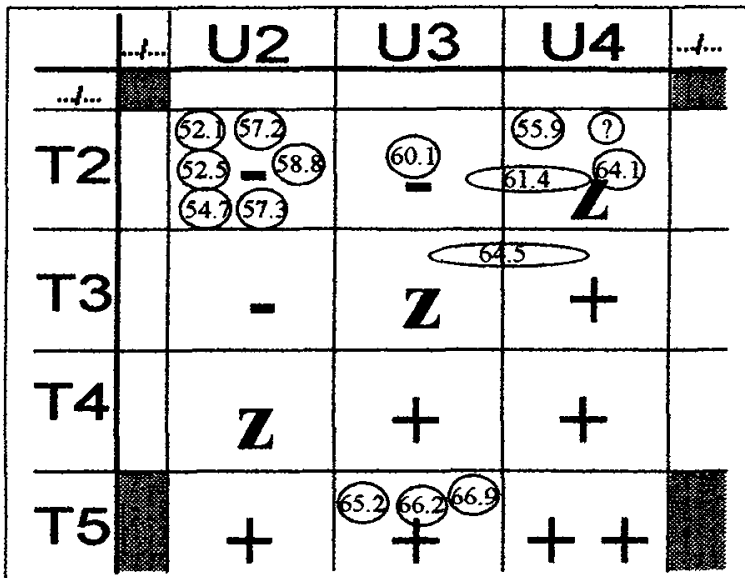

fig. 4 : projection des niveaux sonores mesurés en R3:

Les différents échantillons, de durée variable se positionnent bien, qualitativement, dans les différentes cellules de la grille.

Les niveaux équivalents par classe UiTi sont les suivants :

U2T2: $55.6 \mathrm{~dB}(\mathrm{~A}) \quad \mathrm{U} 3 \mathrm{~T} 2: 60.3 \mathrm{~dB}(\mathrm{~A})$

U4T2: $62.4 \mathrm{~dB}(\mathrm{~A}) \quad$ U3T3: $64.5 \mathrm{~dB}(\mathrm{~A})$

U4T3: $64.5 \mathrm{~dB}(\mathrm{~A}) \quad$ U3T5: $66.2 \mathrm{~dB}(\mathrm{~A})$

On observe bien une augmentation significative du niveau sur la suite U2T2, U3T2 et U4T2, et de U3T2 à U3T5.

Par ailleurs, si on estime le niveau sonore en situation sans effets météo (en U3T3) à environ $64 \mathrm{~dB}(\mathrm{~A})$, on constate bien la non symétrie entre les zones à

renforcement du niveau sonore et les zones à atténuation. Ceci se caractérise par un écart plus faible du niveau en U3T5 par rapport à U3T3. On constate également une plus grande dispersion dans les résultats en zone (-) qu'en zone $(+)$.

Une analyse du récepteur R6 fournit des résultats analogues. Ainsi, le regroupement des $L_{A}$ eq dans chaque classe UiTi fournit les résultats suivants :
U2T2: $54.8 \mathrm{~dB}(\mathrm{~A})$
U3T2: $54.8 \mathrm{~dB}(\mathrm{~A})$
U4T2: $60.1 \mathrm{~dB}(\mathrm{~A})$
U3T3: $64.4 \mathrm{~dB}(\mathrm{~A})$
U4T3: $64.4 \mathrm{~dB}(\mathrm{~A})$
U3T5: $65.2 \mathrm{~dB}(\mathrm{~A})$

En procédant à une analyse identique, pour les récepteurs placés seulement à $320 \mathrm{~m}$ de la source, on constate également que la grille fournit des résultats corrects. Les LAeq de chaque classe UiTi au récepteur R5 (donc à $320 \mathrm{~m}$ et $1,5 \mathrm{~m}$ du sol) sont les suivants :
U2T2: $60.8 \mathrm{~dB}(\mathrm{~A})$
U3T2: $66.1 \mathrm{~dB}(\mathrm{~A})$
U4T2: $68.1 \mathrm{~dB}(\mathrm{~A})$
U3T3: $73.5 \mathrm{~dB}(\mathrm{~A})$
U4T3: $73.5 \mathrm{~dB}(\mathrm{~A})$
U3T5: $76.5 \mathrm{~dB}(\mathrm{~A})$

En estimant à $73 \mathrm{~dB}(\mathrm{~A})$ le niveau sonore sans effets météo (U3T3), on constate comme à $640 \mathrm{~m}$, qu'à mesure que l'on se rapproche d'un vent portant, les écarts à la valeur de référence $73 \mathrm{~dB}(\mathrm{~A})$ diminuent . C'est à dire que l'on constate expérimentalement qu'une situation défavorable pour la propagation (notée - ou - -) produit des niveaux très faibles et (théoriquement) très dispersés, et qu'inversement une situation favorable (notée + ou ++) produit des niveaux peu dispersés et peu supérieurs à une mesure sans effets météo.

La classe U3T5 correspond à une propagation favorisée (notée +). On y observe en effet des niveaux sonores supérieurs à ceux obtenus dans les classes précédemment citées .

Pour le récepteur R2, on observe la même progression sur U2T2 U3T2 et U4T2. L'illustration d'une propagation favorisée, en U3T5 est moins nette qu'en R5. Par contre, on observe très bien que la dispersion des niveaux obtenus est décroissante suivant U2T2, U3T2 et U4T2.

\section{6 - CONCLUSION}

Une expérimentation nous a donc permis de valider l'emploi de cette grille. II est ainsi désormais possible d'en avoir une utilisation qualitative, dans des applications opérationnelles, et de bénéficier de sa grande simplicité d'emploi.

Cette grille va faire l'objet par la suite, d'affinages éventuels, notamment dans les zones extrêmes de compensation (U4T2 et U2T4).

\section{Bibliographie:}

[1] SECHET , GROLLEAU ; Influence des conditions météorologiques sur la propagation du son Institut de Mathématiques Appliquées - Angers - 1992

[2] ZOUBOFF, BERTRAND, BRUNET; Prise en compte qualitative de l'influence des conditions météorologiques dans la propagation du bruit à grande distance

EUROSYMPOSIUM "maitrise du bruit routier en milieu urbain" Mai 1992

[3] ZOUBOFF, BRUNET, BERTRAND, SECHET ; A qualitative approach of meteorological effects on noise propagation - à paraître dans Applied Acoustics 